\title{
A cream containing linoleic acid, 5\% dexpanthenol and ceramide in the treatment of atopic dermatitis
}

\author{
Phatthanan Somjorn, ${ }^{1}$ Nanticha Kamanamool, ${ }^{2}$ Silada Kanokrungsee, ${ }^{1}$ Salinee Rojhirunsakool, ${ }^{1}$ Montree Udompataikul
}

\begin{abstract}
Background: Nowadays, moisturizers contain non-steroidal anti-inflammatory agents that help for treatment of atopic dermatitis (AD). Defensi ${ }^{\circledR}$ (black currant seed oil, sunflower oil, and balloon vine), a new anti-inflammatory, obtained from plant extracts, remain had a few studies for $\mathrm{AD}$.
\end{abstract}

Objective: To compare the effectiveness of moisturizer containing $3 \%$ Defensil ${ }^{\bullet}, 5 \%$ dexpanthenol and ceramide (LDC) with $5 \%$ urea cream in childhood AD treatment.

Methods: Thirty-eight patients with diagnosis of atopic dermatitis by UK working party's criteria were recruited in randomized, controlled, double-blinded 4-week study. The patients were received with twice-daily application of LDC cream on one side of the body and 5\% urea cream on the opposite side. The clinical severity was assessed by modified scoring of atopic dermatitis (SCORAD). Median time to remission was analyzed by survival analysis.

Results: Thirty-seven out of 38 patients accomplished the protocol. The clinical SCORAD significantly improved from baseline in both groups $(p<0.001)$ after 2 and 4 weeks. Furthermore, the LDC group significantly reduced severity of disease better than the $5 \%$ urea group $(P=0.043)$. The mean difference SCORAD scores were $-13.83( \pm 1.83)$ and -13.04 $( \pm 3.22)$ respectively. Stratum corneum hydration $(\mathrm{SCH})$ was enhanced from baseline in both groups $(p<0.001)$ but no statistically significant difference between both groups. Median time to remission had no statistically significant difference $(P=0.697)$.

Conclusion: The effectiveness of LDC cream is better than $5 \%$ urea cream for improving clinical atopic dermatitis. It was suggested that moisturizer containing LDC could be used for the treatment of mild-to-moderate childhood atopic dermatitis.

Key words: atopic dermatitis, linoleic acid, urea cream, Defensil ${ }^{\circledR}$, moisturizer

\footnotetext{
From:

1 Skin Center, Srinakharinwirot University, Bangkok, Thailand

2 Department of Preventive and Social Medicine,

Srinakharinwirot University, Bangkok, Thailand

Corresponding author:

Montree Udompataikul

Skin Center, Srinakharinwirot University, Bangkok, 10110, Thailand

E-mail: umontree@gmail.com
}

$\begin{array}{ll}\text { Abbreviations } \\ \text { AD } & \text { Atopic dermatitis } \\ \text { SCORAD } & \text { Scoring of atopic dermatitis } \\ \text { LDC } & \text { Linoleic acid, dexpanthenol and ceramide } \\ \text { Syndet } & \text { Synthetic detergent } \\ \text { SCH } & \text { Stratum corneum hydration } \\ \text { TEWL } & \text { Transepidermal water loss }\end{array}$

\section{Introduction}

Atopic dermatitis (AD) is a chronic, relapsing and remitting inflammatory skin disorder. Atopic dermatitis commonly flares or exacerbates off and on over the years. The estimated prevalence of $\mathrm{AD}$ among children was $15-30 \%$ and $2-10 \%$ among adults. The onset of $\mathrm{AD}$ mostly occurs within the first year of life, mainly develops during the first six months, and begins before the age of $5 .^{1}$ In addition, $70 \%$ of these children showed that they have clinical improvement and spontaneous remission before becoming adolescence. ${ }^{2}$ Clinical presentation of $\mathrm{AD}$ vary in morphology and distribution of rash depending on age and disease severity. Pediatric patients under two years old are typically affected on the face, neck, and extensor surface of extremities. ${ }^{3}$ After two years old, the rash usually appears on the face, neck, and flexure surface of extremities. The skin lesion manifests as papules, vesicles, 
erythematous lichenified patches or plaques. The skin common skin symptoms are usually dry, and itchy, ${ }^{2}$ impacting the quality of life, sleep disturbance, and school performance. ${ }^{4-6}$

The pathogenesis of $\mathrm{AD}$ includes genetic, immunologic, and environmental factors that can cause the defect of skin barrier functions and allows pathogens, irritants and allergens to penetrate through the skin which produce eczematous lesions. ${ }^{2,3}$ The important risk of $\mathrm{AD}$ lesion increased up to twofold is positive family history. ${ }^{7}$ Filaggrin gene mutation is one of the causes that can lead to the absence of natural moisturizing factors (NMFs) and result in dry skin. Moreover, IgE-mediated the sensitization of food and environmental allergens with skin barrier dysfunction leads to local skin inflammation. ${ }^{8}$

There are many treatments provided for atopic dermatitis such as topical corticosteroid, topical calcineurin inhibitors, systemic corticosteroids, phototherapy, and biologic drugs. ${ }^{3,9,10}$ However, long-term use of corticosteroids associated with adverse effects such as skin atrophy, striae, and hypopigmentation. ${ }^{10,11}$ Therefore, topical moisturizer therapy is preferred and used as adjunctive treatment for all disease severities. ${ }^{12,13}$ Moisturizers help improve skin barrier dysfunction and decrease the frequent use of corticosteroids. ${ }^{14}$

Nowadays, there is an emergent interest in moisturizer containing non-steroidal anti-inflammatory agents from botanical substances, vitamins and minerals that can improve skin inflammation and reduce corticosteroid use. ${ }^{14-17}$ There are many studies about moisturizers containing non-steroidal anti-inflammatory agents in AD. However, their efficacy is usually compared to topical steroids. Few studies are comparing to standard emollients for $\mathrm{AD}$ treatment such as $5 \%$ urea cream. Accordingly, the purpose of the study was to compare the efficacy of moisturizer containing linoleic acid, dexpanthenol and ceramide (Provamed Derma Soothing Cream ${ }^{\oplus}$ NBD, Bangkok, Thailand) to $5 \%$ urea cream for treatment in childhood atopic dermatitis. Defensil ${ }^{\otimes}$ is composed of black currant seed oil, sunflower seed oil and balloon vine extract. Its active ingredients are linoleic acid (extracted from black currant seed oil and sunflower seed oil) and phytosterol (phytosterol extracted from balloon vine).

\section{Method}

\section{Study sizes and population}

This double-blinded, randomized controlled 4-week study includes 38 patients with active atopic dermatitis on both sides. The sample size was calculated by using PS (Power and Sample Size Calculation) program by setting the size of difference $=20 \%$ and the sample correlation coefficient $\mathrm{r}$ (phi) $=$ 0.6 (paired t-test sample size) with $95 \%$ confidence and $90 \%$ power. A sample size of 38 patients was required including a $10 \%$ of drop out.

\section{Recruitment and randomization}

The participants aged 2 to 18 years old with diagnosis of atopic dermatitis by using the UK working party's criteria with mild and moderate diseases were included. Concerning severity grading of SCORAD score, 0-25 scores were defined as mild diseases and 26-50 scores as moderate severity.
We recruited the participants who had equally active atopic dermatitis rash on both sides of their body. It is for controlling the quality of the treatment in the split-body study. A washing out period of up to two weeks was required for the participants who applied the previous moisturizers and topical medications such as topical corticosteroids or calcineurin inhibitors, and four weeks for the participants who received systemic corticosteroid or NSAIDs. Itching control by oral antihistamine was allowed to continue during the period of this study.

The participants who used topical or systemic corticosteroids during the study period and those who lost the following up for 2 times would be excluded from this study. The participants with active skin infection were also excluded. The SCORAD, skin hydration, and patients' satisfactions were measured. The protocol of this study, all participants had to follow up at the 2nd and the 4th week and if the participants who still kept on using the cream but were not able to follow up at an appointment date, they could re-visit in the next 2 weeks.

A computer generated the randomization sequence. The patients received a topical moisturizer containing linoleic acid, 5\% dexpanthenol and ceramide (LDC cream) randomly on one side of their bodies and 5\% urea cream on the other side. The allocation sequence was concealed from all participants and investigators by the third party.

\section{Intervention}

All participants were informed and concealed. They received three products: moisturizing gentle cleanser, cream base (apply at non-atopic areas on both sides) and the test cream (apply on active skin lesions). The containers were labelled as "left" or "right" to apply on atopic areas. Both test creams had the same color, odor, and texture provided in the same containers that we prevented contamination between both creams by informing the participants and their parents how to apply them.

The LDC cream contains three major active ingredients; linoleic acid, 5\% dexpanthenol and ceramide mix containing ceramide 1,3 and 6 . Also, the cream base is composed of butylene glycol, glyceryl stearate, and disodium EDTA. Urea cream consists of $5 \%$ urea with the same cream base formula as LDC cream.

\section{Assessment and outcomes}

Clinical outcomes were assessed by SCORAD, IGA (Investigator Global Assessment Scale) and patients' satisfaction score which was graded from 1 to 5 as poor, no change, fair, good and excellent, respectively. The children under 12 years old were assessed by asking their parents' satisfaction, and the children over 12 years old were given the visual analog scale to evaluate their satisfaction.

Stratum corneum hydration ( $\mathrm{SCH}$ ) was measured with Corneometer $^{\circledast}$ CM 825 (Courage \& Khazaka Electronic $\mathrm{GmbH}$, cologne, Germany).

The remission of clinical outcome was defined as IGA clear or almost clear (IGA $=0$ or 1$).{ }^{18}$ Adverse effects were recorded. This study was approved by the Human Research Ethics Committee of Srinakharinwirot University. All patients 
and their parents were asked to fill out the informed consent form for participating in the study. Investigators who evaluated all outcome measurements were blinded to the treatment allocation. Data were collected three times at baseline, $2^{\text {nd }}$ and $4^{\text {th }}$ week. Clinical trial registration number: TCTR20200826001.

\section{Statistical analysis}

The clinical outcomes of the SCORAD score and IGA were assessed by a mixed-linear model from STATA (version 14). Baseline characteristics were described by mean \pm SD or median (interquartile range) for continuous data, number and percentage for categorical data. Kaplan-Meier analysis for median time to remission and rate of 50\% improvement SCORAD and remission in both groups was used. Mixed-linear model used for assessing the improvement of skin hydration. Intention-to-treat analysis was used for evaluating if there were lost follow-up of patients. The $p$-value of $<0.05$ was considered statistically significant.

\section{Results}

The data were collected from January 2019 to April 2019. The CONSORT flow diagram was illustrated in Figure $\mathbf{1}$.

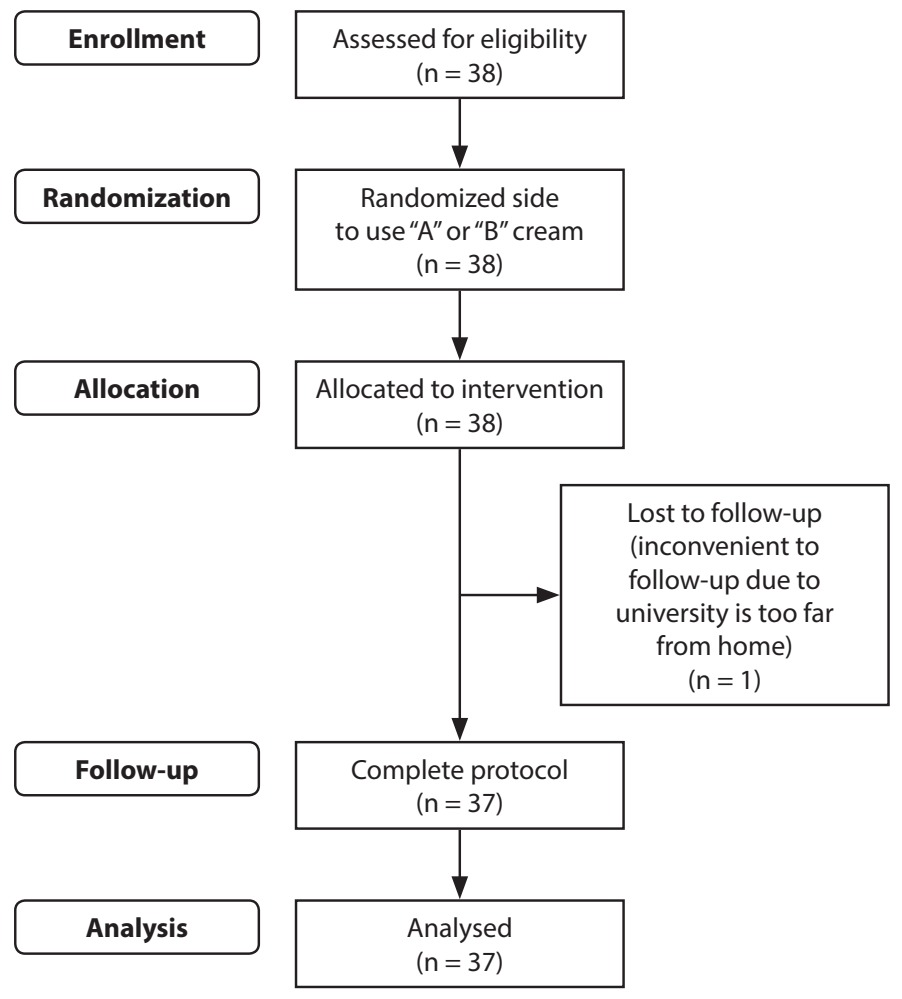

Figure 1. CONSORT flow diagram.

\section{Demographic data}

Thirty-eight participants including 20 males (54.05\%) and 17 females (54.95\%) were enrolled in this study and 37 out of 38 participants completed the protocol. There were 12 $(31.58 \%)$ participants who extended follow up the period of time and collected the data after 4 weeks. However, there was one patient who was excluded due to incomplete follow-up.
The mean age of the participants was 8.5 years old. According to demographic data illustrated in Table 1, there were $31(83.78 \%)$ participants and most of the participants had a family history of atopy. Additionally, 16 (43.24\%) participants used synthetic detergent (syndet) and 32 (86.48\%) participants used moisturizers in their daily routine. The baseline SCORAD score, disease severity and skin hydration (by Corneometer ${ }^{\circledast}$ ) had no statistically significant difference between two groups (Table 2).

Table 1. Demographic data.

\begin{tabular}{|c|c|}
\hline Demographic data & $\begin{array}{l}\text { Patients } \\
(n=37)\end{array}$ \\
\hline \multicolumn{2}{|l|}{ Sex, n (\%) } \\
\hline Female & $17(45.95)$ \\
\hline Male & $20(54.05)$ \\
\hline Age $($ Mean \pm SD $)$ & $8.49 \pm 4.85$ \\
\hline Weight $($ Mean \pm SD) & $31.48 \pm 17.44$ \\
\hline \multicolumn{2}{|l|}{ History of food allergy (\%) } \\
\hline Yes & $13(35.14)$ \\
\hline No & $24(64.86)$ \\
\hline \multicolumn{2}{|l|}{ History of airborne allergy (\%) } \\
\hline Yes & $21(56.76)$ \\
\hline No & $16(43.24)$ \\
\hline \multicolumn{2}{|l|}{ Family history of allergy (\%) } \\
\hline Yes & $31(83.78)$ \\
\hline No & $6(16.22)$ \\
\hline \multicolumn{2}{|l|}{ History of emollients (\%) } \\
\hline No emollient & $5(13.51)$ \\
\hline Use emollients & $32(86.48)$ \\
\hline \multicolumn{2}{|l|}{ Previous type of soaps (\%) } \\
\hline No cleanser & $1(2.70)$ \\
\hline Pure soap & $20(54.05)$ \\
\hline Synthetic detergent & $16(43.24)$ \\
\hline
\end{tabular}

Table 2. Baseline characteristic.

\begin{tabular}{lccc} 
& LDC & $\begin{array}{c}\mathbf{5 \%} \text { urea } \\
\text { cream }\end{array}$ & $\begin{array}{c}\boldsymbol{P} \\
\text { value }\end{array}$ \\
$\begin{array}{l}\text { Baseline SCOARD score } \\
\text { (Mean } \pm \text { SD) }\end{array}$ & $32.79 \pm 8.31$ & $34.57 \pm 8.97$ & 0.114 \\
$\begin{array}{l}\text { Baseline of disease severity (\%) } \\
\text { Mild }\end{array}$ & $24.32 \%$ & $21.26 \%$ & 0.782 \\
$\begin{array}{l}\text { Moderate } \\
\text { Itch score }\end{array}$ & $75.68 \%$ & $78.38 \%$ & \\
$\begin{array}{l}\text { Baseline skin hydration (by } \\
\text { Corneometer }) \text { (Mean } \pm \text { SD) }\end{array}$ & $28.84 \pm 15.79$ & $31.23 \pm 16.50$ & 0.355 \\
\hline
\end{tabular}




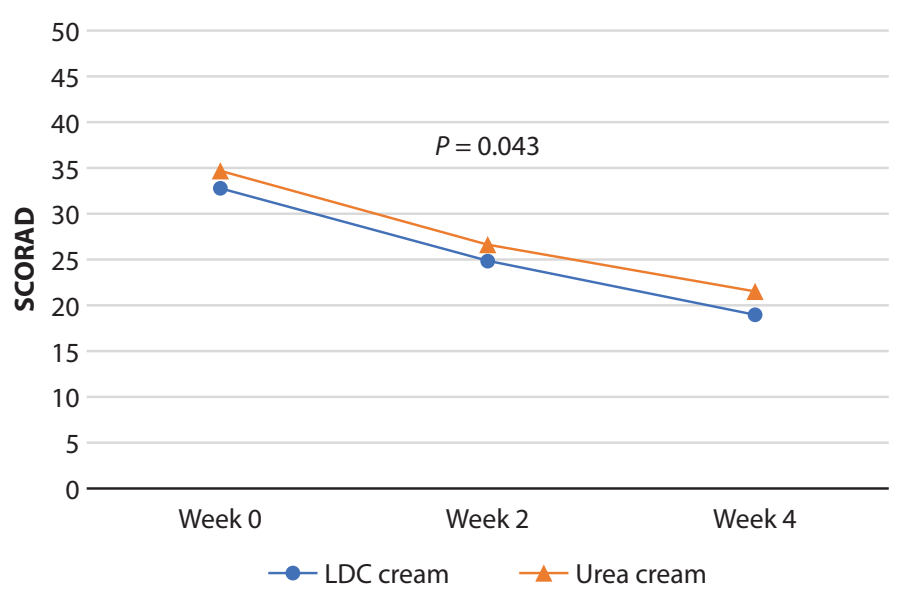

Figure 2. The SCORAD score improvement of atopic dermatitis.

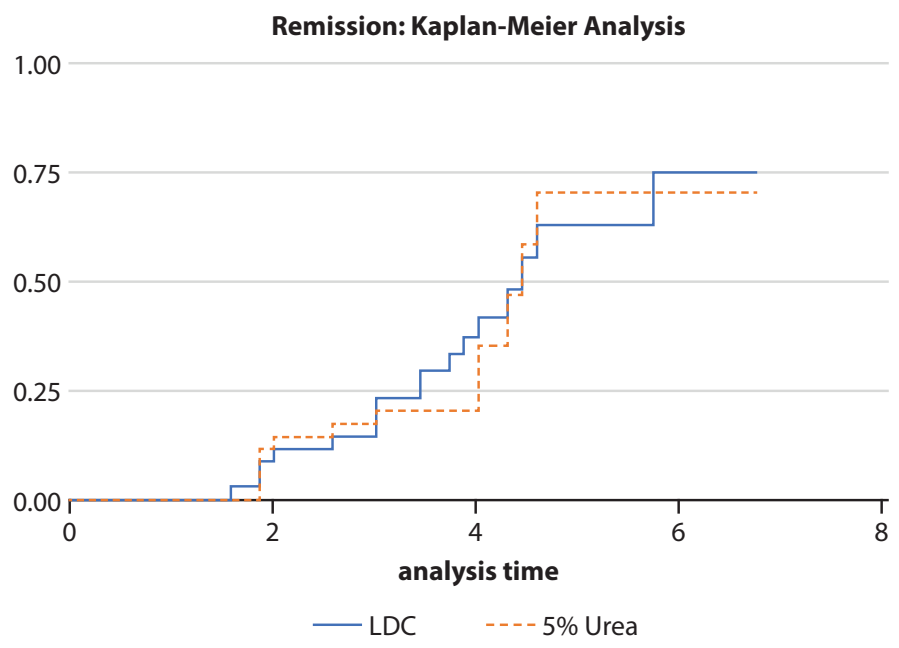

Figure 3. The median time to remission of atopic dermatitis participants analyzed by survival analysis (STATA version 14).
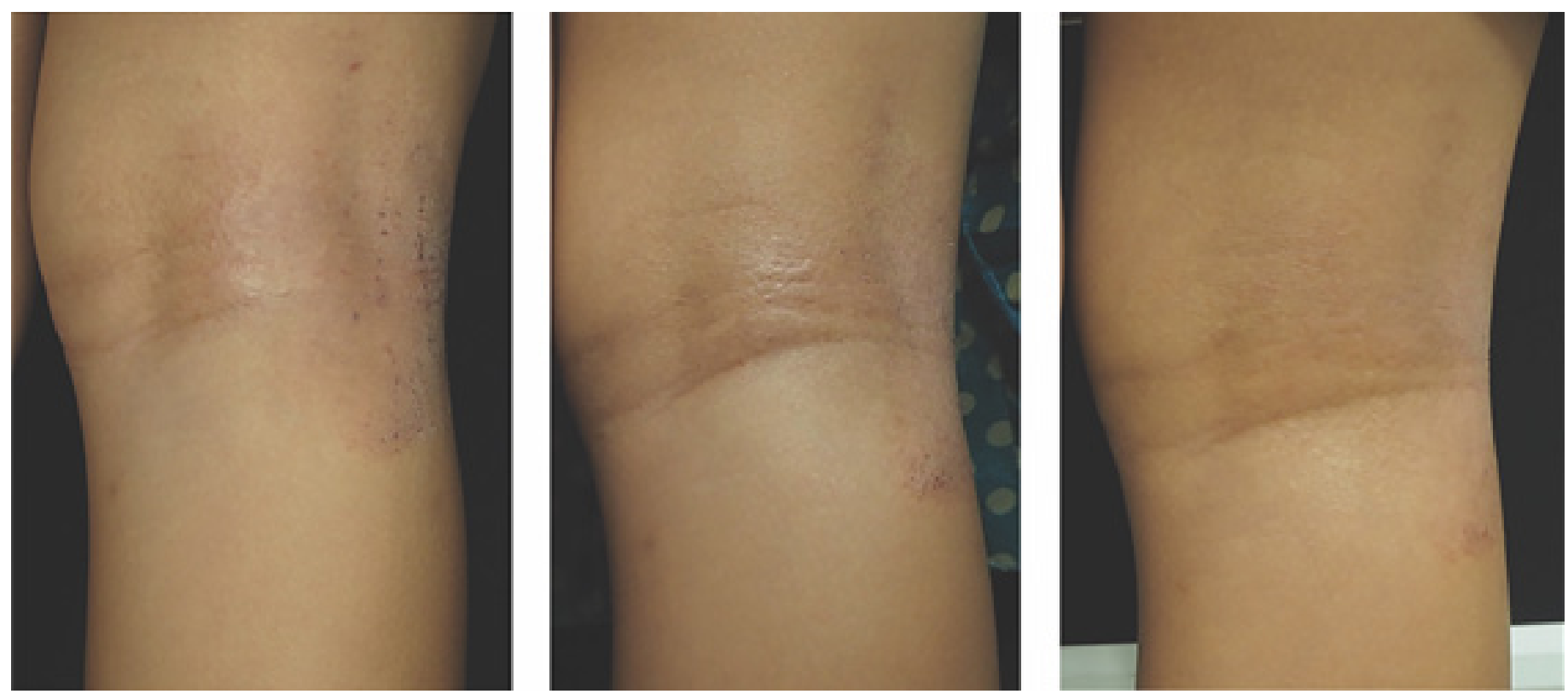

Figure 4. The clinical response of the atopic dermatitis participant in the LDC cream group at baseline at the $2^{\text {nd }}$ week and the $4^{\text {th }}$ week.
Improvement of SCORAD score and remission of atopic dermatitis were the primary outcomes of this study. The mean value of baseline SCORAD score of LDC treatment side and urea treatment side was 32.79 and 34.57 respectively without statistically significant difference $(P=0.114)$ as shown in Table 2. Both sides showed improvement of SCORAD score significantly from baseline $(P<0.001)$. Furthermore, the reduction SCORAD score of the LDC group decreases more significantly than the $5 \%$ urea group $(P=0.043)$ as shown in Figure 2. There were $14(37.83 \%)$ and $13(35.14 \%)$ participants in the LDC and the urea groups achieved 50\% improvement SCORAD respectively.

The median time to reach $50 \%$ improvement SCORAD of both groups was 4.43 weeks (95\% CI 0.56-2.34). After analyzing by using a survival analysis program, we found that the $50 \%$ improvement SCORAD of LDC group tended to be higher than the urea group about 14\% nevertheless there was no statistically significant difference [HR of LDC/urea group was 1.14] $(P=0.713)$.

The median time to remission of both groups was 4.28 weeks (95\% CI $0.58-2.26)$ as in Figure 3. There were 17 (44.74\%) and $16(42.11 \%)$ participants who achieved remission criteria in the LDC group and the 5\% urea group, consecutively. Survival analysis of remission showed that the LDC group tended to reach remission higher than the 5\% urea group about $14 \%$, however, there was no statistically significant difference [HR of LDC/urea group was 1.14] $(P=0.697)$.

The secondary outcomes in the study were SCH (by using Corneometer ${ }^{\circledR}$ ), patients' satisfaction and side effects. The mean value of baseline skin hydration of the LDC and the 5\% urea group was 28.84 and 31.23 , respectively, with no statistically significant difference $(P=0.355)$, as shown in Table 2. After 4-week of treatment, the SCH of the LDC and urea group was 51.28 and 46.60 , respectively with statistical significance from baseline $(P<0.001)$. The mean difference of 

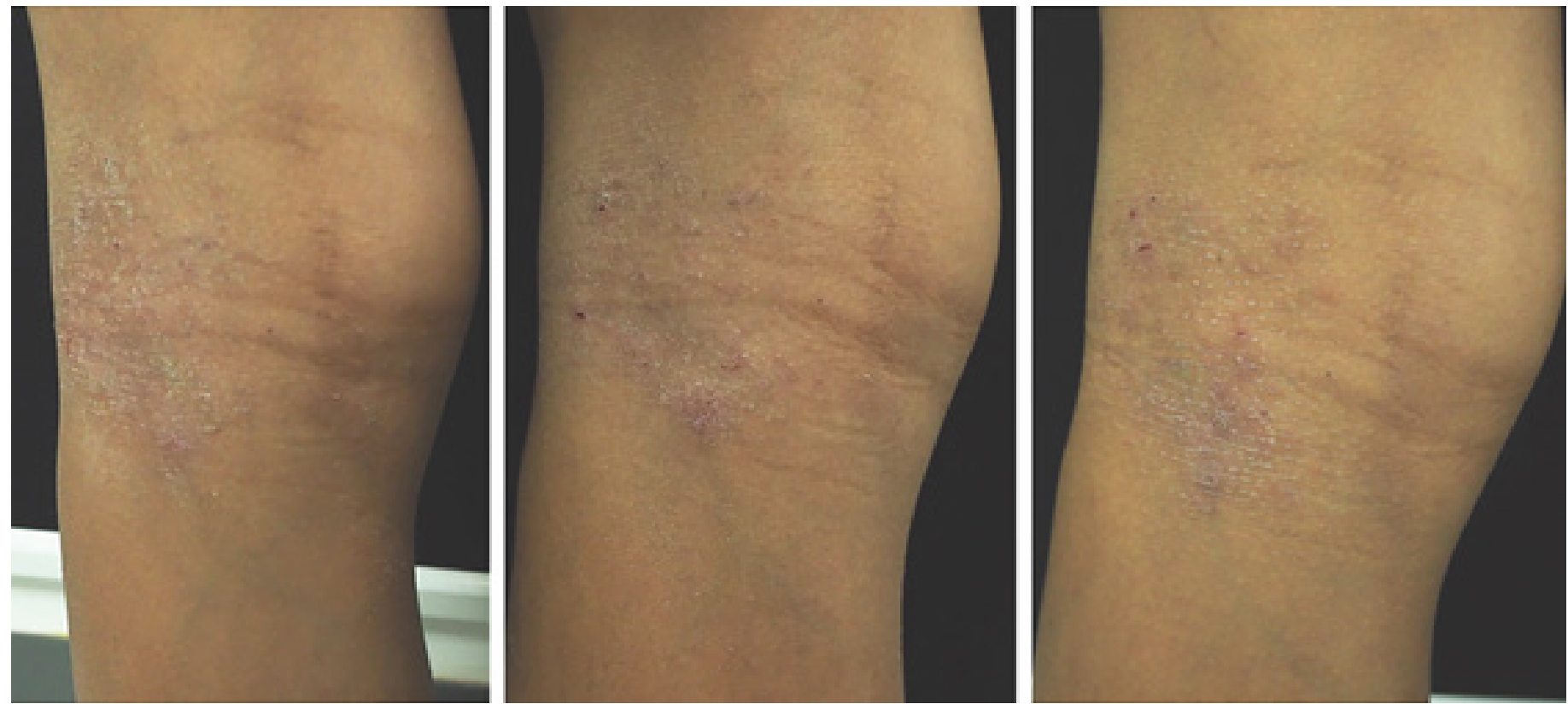

Figure 5. The clinical response of the atopic dermatitis participant in the urea cream group at baseline at the $2^{\text {nd }}$ week and the $4^{\text {th }}$ week.

skin hydration improvement of the LDC and the urea group was 23.44 and 15.37 , respectively with no statistical significance between groups $(P=0.139)$.

With regard to patients' satisfaction, it was found that $29.72 \%$ of the LDC group gave the excellent score (5/5) and $27.03 \%$ in the urea group gave the same score. The average score of the LDC group and the urea group was $3.73 \pm 1.15$ and $3.54 \pm 1.37$, respectively, with no statistical difference between groups $(P=0.360)$. The clinical response pictures were demonstrated in Figure 4 and 5.

Finally, it was shown that the LDC group's side effect was $8(21.62 \%)$ and the 5\% urea group was $12(32.43 \%)$, respectively. The side effect from the $5 \%$ urea group was higher than the LDC group. However, there was no statistical group difference $(P=0.295)$. Mild irritation (transient stinging) was $8(21.62 \%)$ and $10(27.02 \%)$ participants in the LDC and the urea group, respectively. In addition, 2 (5.41\%) participants had slightly moderate irritation in the $5 \%$ urea group as the severity was related with the participants who had fissures on AD skin lesions.

\section{Discussion}

The recent research on the treatment of atopic dermatitis focused on the correction of skin barrier dysfunction, one of the main pathophysiology of $\mathrm{AD} .{ }^{19}$ The loss or dysfunction of the filaggrin gene leads to a decrease in filaggrin protein which is a corneocyte envelope protein that converts to natural moisturizing factors (NMFs). ${ }^{20,21}$ NMFs contains significant substance, for example, polycarboxylic acid, lactic acid and urea. It acts as a humectant for skin hydration. ${ }^{22,23}$ Hence, the lack of filaggrin protein results in skin dryness. Furthermore, it raises the skin $\mathrm{pH}$ stimulating serine protease and initiating skin inflammation consequently. ${ }^{19}$

Additionally, AD patients had reduced epidermal intercellular lipid components, it was a so-called ceramide especially ceramide I and III. ${ }^{24,25}$ Ceramide is an occlusive moisturizer that helps increase the skin hydration by preventing the transepidermal water loss (TEWL) from the skin. ${ }^{25,26}$ Another crucial intercellular lipid is linoleic acid, an essential fatty acid of the skin, which plays an important role in anti-inflammatory process in $\mathrm{AD}$ as well. Actually delta-6-desaturase change linoleic acid to gamma linoleic acid (GLA). ${ }^{24}$ GLA rapidly converts to dihomo-gamma-linoleic acid, the precursor of Prostaglandin $\mathrm{H} 1$, and subsequently produces anti-inflammatory agents such as prostaglandin E1 (PGE1) and thromboxane A1 (TXA1). ${ }^{27,28}$ AD patients have a low level of this enzyme. Therefore, they have low levels of PGE1 and TXA1, accordingly, the skin is easy to have inflammation aggravated by antigens. Lastly, this study also showed that both LDC cream and $5 \%$ urea cream could effectively be used in the remission phase.

The linoleic acid depletion in $\mathrm{AD}$ skin plays a major role in the pathophysiology of this disease. ${ }^{29}$ A previous study showed that moisturizer containing linoleic acid and 5\% urea cream cloud reduces the SCORAD score in AD patients significantly different from the baseline. Nevertheless, there was no significant group difference. ${ }^{30}$ The study results contradict to our study, which demonstrated that moisturizer containing linoleic acid, dexpanthenol and ceramide could reduce the clinical features by SCORAD score more than 5\% urea cream. Though the skin hydration and the patients' satisfaction with both creams were not significantly different.

It can be explained that not only linoleic acid but also phytosterol, ceramide and dexpanthenol play an additive role in the treatment. Phytosterol had anti-inflammatory effect. ${ }^{31}$ Dexpanthenol or pantothenic (vitamin B5) possess humectant moisturizing activity and anti-inflammatory effect. Besides, dexpanthenol, a component of coenzyme A, is important for metabolism of carbohydrate, fatty acid, hormone and protein. As a result, it promotes wound healing. An in vitro study 
demonstrated the benefit of dexpanthenol on human fibroblast to improve cell proliferation and wound healing. Regarding the clinical study which supports its efficacy showed that $5 \%$ dexpanthenol water-in-oil formulation had the same efficacy as $1 \%$ hydrocortisone in the treatment of mild to moderate $\mathrm{AD} .{ }^{14}$

The role of moisturizer in skin repair has been used as a fundamental and standard therapy for all severity of $\mathrm{AD}$. It restores the barrier function by improving skin hydration through occlusive and humectant mechanisms. The recent novel of moisturizer demonstrated by many studies concludes that the moisturizer with non-steroidal anti-inflammatory agents could be used as an alternative treatment for mild to moderate AD. ${ }^{14,30}$ Our present study is an updated trial supporting this conclusion. Firstly, the clinical lesions are improved via the anti-inflammatory effect of linoleic acid, phytosterol, and dexpanthenol. Secondly, the barrier dysfunction was replenished by the occlusive action of linoleic acid, ceramide, and humectant effect of both dexpanthenol and glycol (from cream base ingredient). Finally, dexpanthenol can promote the wound healing process of the lesions.

According to systematic review, it was demonstrated that moisturizers ingredients for example glycerin, propylene glycol, ceramide etc. including urea showed the beneficial outcomes on clinical severity, transepidermal water loss (TEWL) and SCH of atopic skin which can be improved by using only moisturizer, moreover, two studies revealed that urea was superior to glycerin for the treatment of $\mathrm{AD}$ and it was suggested to use as first choice of emollient for AD. ${ }^{32}$ Urea has a humectant effect that helps promote stratum corneum hydration as a result it improves skin barrier function which is the main pathogenesis of atopic dermatitis. On the contrary, LDC cream had both anti-inflammatory effect and hydration effect, so it could improve clinical SCORAD from baseline and reduced clinical severity superior to the urea cream group significantly.

The limitation of this study might be a short period of follow-up (4 weeks). The next investigation with a longer period of follow up after remission (in maintenance phase) might reveal a different outcome comparing the two formulations. The amount of cream that the participants apply on lesions should be measured in $\mathrm{mg} / \mathrm{cm}^{2}$ because the amount of cream can affect the result. Moreover, objective measurements such as TEWL and skin conductance were not evaluated in this study. It should be evaluated in further study.

\section{Conclusion}

In conclusion, the LDC cream's effectiveness is better than $5 \%$ urea cream due to the clinical improvement of skin lesions in mild to moderate atopic dermatitis. It was suggested that moisturizers containing LDC could be used as an alternative treatment for acute and maintenance phases in mild-to-moderate childhood AD.

\section{Acknowledgements}

The author would like to thank Dr. Wattinee Amornpet$\mathrm{kul}$ for the role of third party in allocation and concealment process. Mr. Anand Paichitrojjana for helping with data collection. Dr. Wachira Suppiboonpul for helping with graphic design.

\section{Disclosure}

All products in this research such as LDC cream, 5\% urea cream, cream base and bath lotion were provided by NBD HEALTHCARE CO., LTD. However, the author declared that the company was not involved in any step of the study.

\section{Funding}

This study received a research fund from NBD HEALTHCARE CO., LTD.

\section{Author contributions}

- Somjorn P. designed and performed the study and wrote the manuscript (participants recruitment, photography, data collection, and interpretation).

- Kamanamool N. performed statistical analysis, discussed the results and approved the manuscript.

- Kanokrungsee S. and Rojhirunsakool S. helped approved the protocol and manuscript.

- Udompataikul M. supervised the project, discussed the result and approved the manuscript.

\section{References}

1. Weidinger S, Novak N. Atopic dermatitis. Lancet. 2016;387(10023): 1109-22.

2. Bieber T. Atopic dermatitis. Ann Dermatol. 2010;22(2):125-37.

3. Thomsen SF. Atopic dermatitis: natural history, diagnosis, and treatment. ISRN Allergy. 2014;2014:354250.

4. Silverberg JI, Gelfand JM, Margolis DJ, Boguniewicz M, Fonacier L, Grayson MH, et al. Patient burden and quality of life in atopic dermatitis in US adults: A population-based cross-sectional study. Ann Allergy Asthma Immunol. 2018;121(3):340-7.

5. Raznatovic Durovic M, Jankovic J, Tomic Spiric V, Relic M, Sojevic Timotijevic Z, Cirkovic A, et al. Does age influence the quality of life in children with atopic dermatitis? PLoS One. 2019;14(11):e0224618.

6. Koszoru K, Borza J, Gulacsi L, Sardy M. Quality of life in patients with atopic dermatitis. Cutis. 2019;104(3):174-7.

7. Dold S, Wjst M, von Mutius E, Reitmeir P, Stiepel E. Genetic risk for asthma, allergic rhinitis, and atopic dermatitis. Archives of disease in childhood. 1992;67(8):1018-22.

8. Turner KJ, Rosman DL, O'Mahony J. Prevalence and familial association of atopic disease and its relationship to serum IgE levels in 1,061 school children and their families. Int Arch Allergy Appl Immunol. 1974;47(5): 650-64.

9. Hajar T, Gontijo JRV, Hanifin JM. New and developing therapies for atopic dermatitis. An Bras Dermatol. 2018;93(1):104-7.

10. Wong ITY, Tsuyuki RT, Cresswell-Melville A, Doiron P, Drucker AM. Guidelines for the management of atopic dermatitis (eczema) for pharmacists. Can Pharm J (Ott). 2017;150(5):285-97.

11. Callen J, Chamlin S, Eichenfield LF, Ellis C, Girardi M, Goldfarb M, et al. A systematic review of the safety of topical therapies for atopic dermatitis. Br J Dermatol. 2007;156(2):203-21.

12. Eichenfield LF, Tom WL, Berger TG, Krol A, Paller AS, Schwarzenberger $\mathrm{K}$, et al. Guidelines of care for the management of atopic dermatitis: section 2. Management and treatment of atopic dermatitis with topical therapies. J Am Acad Dermatol. 2014;71(1):116-32. 
13. Ring J, Alomar A, Bieber T, Deleuran M, Fink-Wagner A, Gelmetti C, et al Guidelines for treatment of atopic eczema (atopic dermatitis) part I. J Eur Acad Dermatol Venereol. 2012;26(8):1045-60.

14. Udompataikul M, Limpa-o-vart D. Comparative trial of $5 \%$ dexpanthenol in water-in-oil formulation with $1 \%$ hydrocortisone ointment in the treatment of childhood atopic dermatitis: a pilot study. J Drugs Dermatol. 2012;11(3):366-74

15. M U. New innovation of moisturizers containing non-steroidal anti -inflammatory agents for atopic dermatitis. . World J Dermatol 2015 2015;4(2): 108-113.

16. Belloni G, Pinelli S, Veraldi S. A randomised, double-blind, vehicle -controlled study to evaluate the efficacy and safety of MAS063D (Atopiclair) in the treatment of mild to moderate atopic dermatitis. Eur J Dermatol. 2005;15(1):31-6

17. Hon KL, Kung JSC, Ng WGG, Leung TF. Emollient treatment of atopic dermatitis: latest evidence and clinical considerations. Drugs Context. 2018;7:212530

18. Papp KA, Werfel T, Fölster-Holst R, Ortonne JP, Potter PC, de Prost Y, et al. Long-term control of atopic dermatitis with pimecrolimus cream $1 \%$ in infants and young children: a two-year study. J Am Acad Dermatol. 2005;52(2):240-6.

19. Kim BE, Leung DYM. Significance of Skin Barrier Dysfunction in Atopic Dermatitis. Allergy Asthma Immunol Res. 2018;10(3):207-15.

20. Tsakok T, Woolf R, Smith CH, Weidinger S, Flohr C. Atopic dermatitis: the skin barrier and beyond. Br J Dermatol. 2019;180(3):464-74.

21. Zaniboni MC, Samorano LP, Orfali RL, Aoki V. Skin barrier in atopic dermatitis: beyond filaggrin. An Bras Dermatol. 2016;91(4):472-8

22. Verdier-Sevrain S, Bonte F. Skin hydration: a review on its molecular mechanisms. J Cosmet Dermatol. 2007;6(2):75-82.

23. Sator PG, Schmidt JB, Honigsmann H. Comparison of epidermal hydration and skin surface lipids in healthy individuals and in patients with atopic dermatitis. J Am Acad Dermatol. 2003;48(3):352-8.
24. Manku MS, Horrobin DF, Morse N, Kyte V, Jenkins K, Wright S, et al. Reduced levels of prostaglandin precursors in the blood of atopic patients: defective delta-6-desaturase function as a biochemical basis for atopy. Prostaglandins Leukot Med. 1982;9(6):615-28.

25. Di Nardo A, Wertz P, Giannetti A, Seidenari S. Ceramide and cholesterol composition of the skin of patients with atopic dermatitis. Acta Derm Venereol. 1998;78(1):27-30.

26. Hon KL, Leung AK. Use of ceramides and related products for childhood-onset eczema. Recent Pat Inflamm Allergy Drug Discov. 2013; $7(1): 12-9$.

27. Horrobin DF. Essential fatty acid metabolism and its modification in atopic eczema. Am J Clin Nutr. 2000;71(1 Suppl):367s-72s.

28. Ikai K, Imamura S. Role of eicosanoids in the pathogenesis of atopic dermatitis. Prostaglandins, Leukotrienes and Essential Fatty Acids. 1993; 48(6):409-16

29. Yang Q, Liu M, Li X, Zheng J. The benefit of a ceramide-linoleic acid-containing moisturizer as an adjunctive therapy for a set of xerotic dermatoses. Dermatol Ther. 2019;32(4):e13017.

30. Nasrollahi SA, Ayatollahi A, Yazdanparast T, Samadi A, Hosseini H, Shamsipour M, et al. Comparison of linoleic acid-containing water-in -oil emulsion with urea-containing water-in-oil emulsion in the treatment of atopic dermatitis: a randomized clinical trial. Clin Cosmet Investig Dermatol. 2018;11:21-8.

31. Babu KCV, Krishnakumari S. Anti-inflammatory and antioxidant compound, rutin in cardiospermum halicacabum leaves. Ancient science of life. 2005;25(2):47-9.

32. Lindh JD, Bradley M. Clinical Effectiveness of Moisturizers in Atopic Dermatitis and Related Disorders: A Systematic Review. Am J Clin Dermatol. 2015;16(5):341-59. 